\title{
Longitudinal vibrations of a cylindrical bar from the action of harmonic forces
}

\author{
Abdukakhkhar Abduvaliev ${ }^{1}$, and Abdulaziz Abdulkhayzoda ${ }^{2 *}$ \\ ${ }^{1}$ Tashkent Institute of Irrigation and Agricultural Mechanization Engineers, Tashkent, Uzbekistan \\ ${ }^{2}$ Tashkent Transport University, Tashkent, Uzbekistan
}

\begin{abstract}
The longitudinal vibration of a cylindrical rod has been studied in an elastic medium from the action of axial harmonic forces in a state of compression tension. The consideration took into account the damping of vibration of the rod according to the theory of E.S. Sorokin. Taking into account, internal friction made it possible to approach the real state of the rod as a source of wave radiation. The amplitude-frequency characteristic of the structure is built, taking into account changes in the parameters of the environment and the rod. The possibilities of the emergence of the resonance state of the rod are established. At the same time, cases were considered with and without inelastic properties of the rod material. The analysis of the influence of the properties of the environment and the rod on the amplitude of the displacement of the latter is carried out.
\end{abstract}

\section{Introduction}

Wave problems are relevant for underground structures, so transverse vibrations of an underground cylindrical structure and vibrations of an elastic half-space with a cylindrical cavity under the action of Rayleigh waves are considered in [1,2], and the vibrations of a hard disk in elastic space are studied in [3].

To reduce the movement of structures, dynamic vibration dampers are used. The possibility of damping vibrations of underground pipelines from the action of Rayleigh waves using a single-mass damper and damping of vibrations of an underground structure using a three-mass damper is considered in $[4,5]$.

In [6], an assessment was made of the stress-strain state of earth dams, taking into account the nonlinear deformation of the material and large deformations, and in [7], an assessment of the dynamic behavior of the "structure - foundation" system was carried out taking into account the wave energy removal. In works $[8,9,10,11]$, dynamic problems for the "structure-soil" system are considered. Nonlinear vibrations of an axisymmetric body under the action of impulse loads, forced axisymmetric vibrations of a viscoelastic cylindrical shell, natural vibrations of structurally inhomogeneous multiply connected shell structures with viscoelastic elements are considered $[12,13,14]$. The method of compensating loads applied to the problems of equilibrium, vibrations and stability of plates and membranes is presented in [15].

*Corresponding author: Abdulaziz_777_7@mail.ru 
Taking into account inelastic properties in dynamic problems allows one to study the real behavior of the system under consideration. Oscillations of mechanical systems taking into account the imperfect elasticity of the material and the effect of viscosity on the propagation of stress waves in elastic bodies under dynamic loads are considered in [16, 17], and in $[18,19]$, the method of taking into account the inelastic resistance of materials when calculating structures for vibrations is studied.

In [20], the dynamic interaction of an embedded cylindrical rod under the action of axial harmonic forces was considered.

\section{Methods}

Consider the longitudinal vibrations of an extended cylindrical rod located in an elastic space from the action of axial harmonic forces.

The differential equation of motion of the rod has the form

$$
E^{*} F \frac{d^{2} u_{p}}{d z^{2}}+2 \pi a \tau_{r z}-\rho F \frac{d^{2} u_{p}}{d t^{2}}=-\frac{2 F_{0}}{L} \sum_{m=1}^{\infty} \cos \alpha_{m} z e^{-i \omega t}
$$

where, $u_{p}(z, t)$ is longitudinal movement of the rod;

$E^{*}$ is complex Young's module $E^{*}=E_{0}(1+i \delta)$;

$\delta$ is parameter that takes into account attenuation in the bar;

$\mathrm{F}, \mathrm{a}, \rho$ is cross-sectional area and radius, bar specific gravity;

$\tau_{r z}$ is shear stress of the medium at the line of contact with the rod.

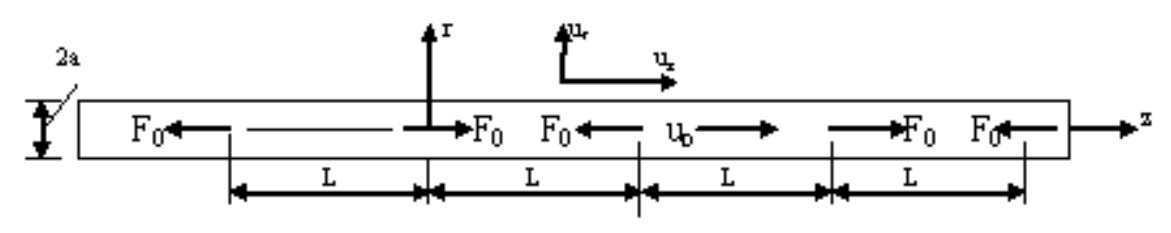

Fig. 1. Design scheme of an underground structure

To solve the problem, we use the results of [20] and omitting intermediate calculations; we present the expression for the longitudinal displacement of the rod in the form

$$
\begin{gathered}
u_{p}=\sum_{m=1}^{\infty} \frac{\frac{2 F_{0} a}{F L} \cos \alpha_{m} z e^{-i \omega t}}{(2 m-1)^{2}(1+i \delta)-\alpha^{2}\left\{1-\frac{2 \gamma p m}{\Omega\left[\gamma_{s m}^{2}+(2 m-1)^{2}(2 \pi \eta)^{2}\right\}}\right]^{\prime}}, \\
\gamma_{p m}=2 \pi \eta(2 m-1)\left[\frac{\alpha^{2}}{(2 m-1)^{2} R_{v}^{2}}-1\right]^{\frac{1}{2}} ; \quad \gamma_{s m}=2 \pi \eta(2 m-1)\left[\frac{\alpha^{2} R_{c}^{2}}{(2 m-1)^{2} R_{v}^{2}}-1\right]^{\frac{1}{2}} \\
\vartheta^{2}=\frac{E_{0}}{\rho}, k^{2}=\frac{\omega^{2}}{\vartheta^{2}}, \alpha=\frac{k L}{\pi}, \eta=\frac{a}{2 L}, R_{v}^{2}=\frac{c_{1}^{2}}{\vartheta^{2}}, R_{c}=\frac{c_{1}}{c_{2}}
\end{gathered}
$$

Assuming (1) to be homogeneous, we obtain the frequency equation, from which we can determine the resonance frequencies

$$
(2 m-1)^{2}(1+i \delta)+\frac{R_{v}^{2} 2 \gamma_{p m}}{R_{c}^{2} \Omega(2 \pi \eta)^{2}(2 m-1)^{2} \Delta_{m}}-\alpha^{2}=0,
$$

where,

$$
\Delta_{m}=\frac{H_{0}^{1}\left(\gamma_{p m}\right)}{H_{1}^{1}\left(\gamma_{p m}\right)}+\frac{\gamma_{p m} \gamma_{s m} H_{0}^{1}\left(\gamma_{s m}\right)}{(2 \pi \eta)^{2}(2 m-1)^{2} H_{1}^{1}\left(\gamma_{s m}\right)}
$$


If we assume that there is no soil $\left(\rho_{0}=0\right)$ and at $\delta=0$, from (4) we get $\alpha_{n}=(2 n-1)$.

From here

$$
\omega_{n}=(2 n-1) \frac{\pi}{L} \sqrt{\frac{E_{0}}{\rho}},(\mathrm{n}=1,2,3, \ldots)
$$

which corresponds to the classical expression for determining the frequencies of natural longitudinal vibrations of the rod.

\section{Results and Discussion}

The main parameters are $\Omega, \eta, R_{v}, \alpha$, on which the amplitude of displacement of the rod (pipeline) depends. These dimensionless quantities are the ratio of the same parameters of the structure and the soil, meaning how many times the parameter of the structure is greater (or less) than the parameter of the soil; is the dimensionless relative frequency of exposure. $\alpha$

Consider the effect of changing the frequency of exposure to vibrations of the structure. Changing $0 \leq \alpha \leq 10$, we will build the amplitude-frequency characteristic (AFC) of the structure. In this case, the quantities $\Omega, \eta, R_{v}$ will be fixed. Let us compare separately the cases $\delta=0$ and $\delta=0,2$.

The parameter $\gamma_{p m}$ characterizing the longitudinal wave has zero values at $\alpha_{m}=$ $(2 m-1) R_{v}$. In this case, the denominator of formula (2) takes the form

$$
(2 m-1)^{2}\left[(1+i \delta)-R_{v}\right]
$$

For a structure that does not have damping $(\delta=0)$, this expression will take the form

$$
(2 m-1)^{2}\left(1-R_{v}\right) \text {. }
$$

For the movement of the structure to be infinite $u_{p}=\infty$, it is necessary to assume $R_{v}=1$. Therefore, the structure will receive infinite movement in the case $\mathrm{c}_{1}=\vartheta$ and at frequencies $\alpha_{m}=(2 m-1)$ (Fig. 3). A structure that has an inelastic property $(\delta=0,2)$ will not receive infinite or too much displacement in any resonance state. Only when the frequency of the impact and the first natural vibration frequency of the structure are equal, the movement of the latter increases noticeably (Fig. 2-4). Resonance is not observed at other frequencies.

Thus, the use of materials with strong damping properties for underground structures will protect it from excessively large resonant vibrations. When dynamically calculating a system with distributed parameters, in addition to determining the frequency equation, as a rule, a graphical dependence of frequencies on the quantities included in this equation is established. This approach lends clarity to the analysis.

In fig. 2 - 4 show the frequency response of the system, taking into account the attenuation in the beam at $\delta=0,2$. The shaded part of the graphs corresponds to the case when $\delta=0$.

\section{Conclusions}

In the construction of underground transport structures made of reinforced concrete, the use of various aggregates is practiced to reduce the structure's weight. Such fillers reduce the 
elastic properties of the structure by up to $30 \%$ and significantly increase the inelastic properties of the building material.

When comparing the graphs with damping $(\delta=0,2)$ and without damping $(\delta=0)$, it can be seen that with an increase in dissipation, the resonant amplitude of the oscillations decreases significantly. If at $\delta=0,2$ (Fig. 4a) several resonances with infinite peaks are observed, then at $\delta=0$ (Fig. $4 \mathrm{~b}$ ) there is one resonance with a finite peak.

Note that the amplitude of displacements outside the resonance zone is insignificant. Thus, it is necessary to protect the structure by preventing it from entering the resonance zone.

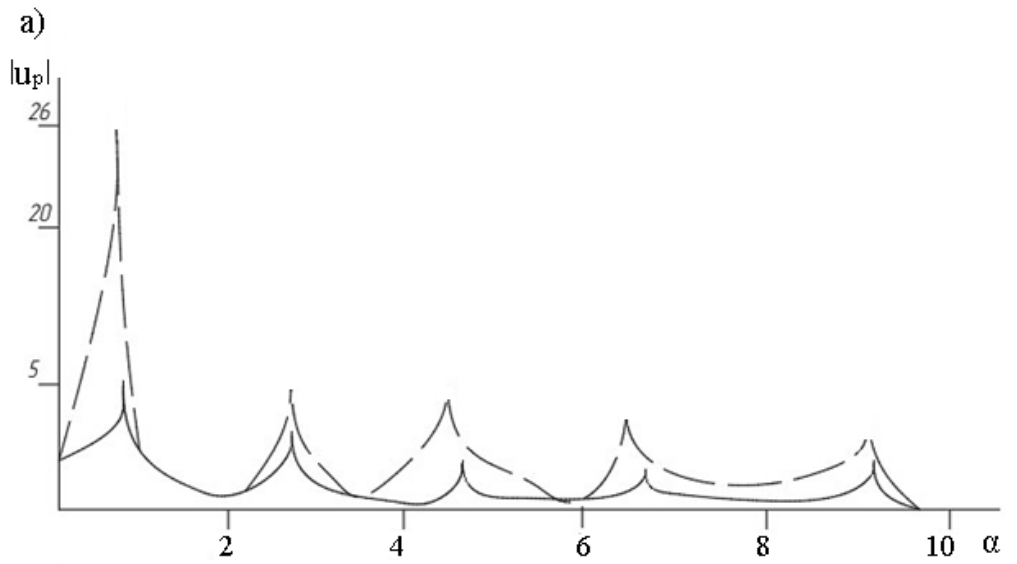

b)

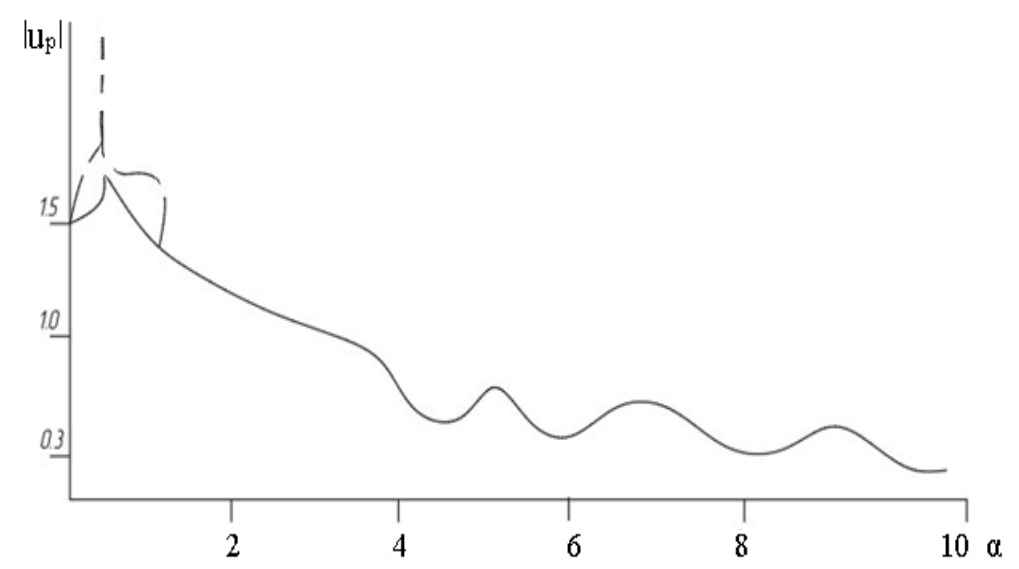

Fig. 2. Amplitude-frequency characteristic of the rod at $\Omega=5 ; \delta=0.2 ; \mathrm{a}-\eta=0.01 ; \mathrm{b}-\eta=0.10$; $R_{v}=0.5$. 

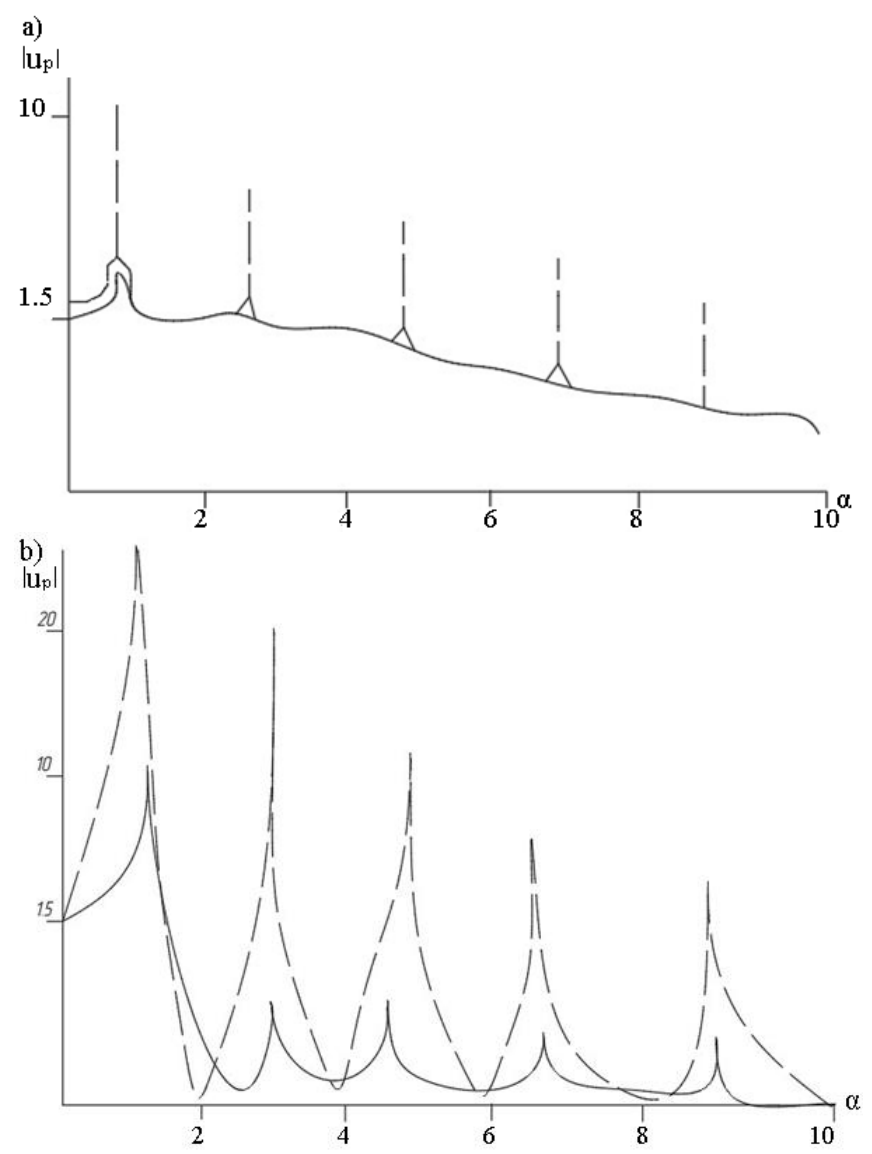

Fig. 3. Amplitude-frequency characteristic of the $\operatorname{rod}$ at $\Omega=5 ; \delta=0.2$; a $-\eta=0.01 ; b-\eta=0.10$; $R_{v}=1$;

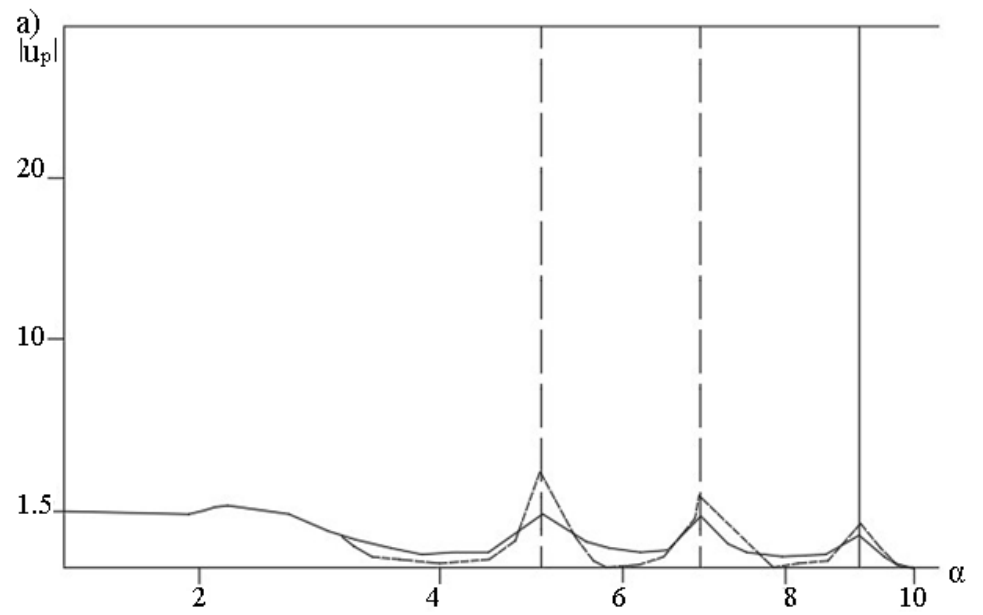




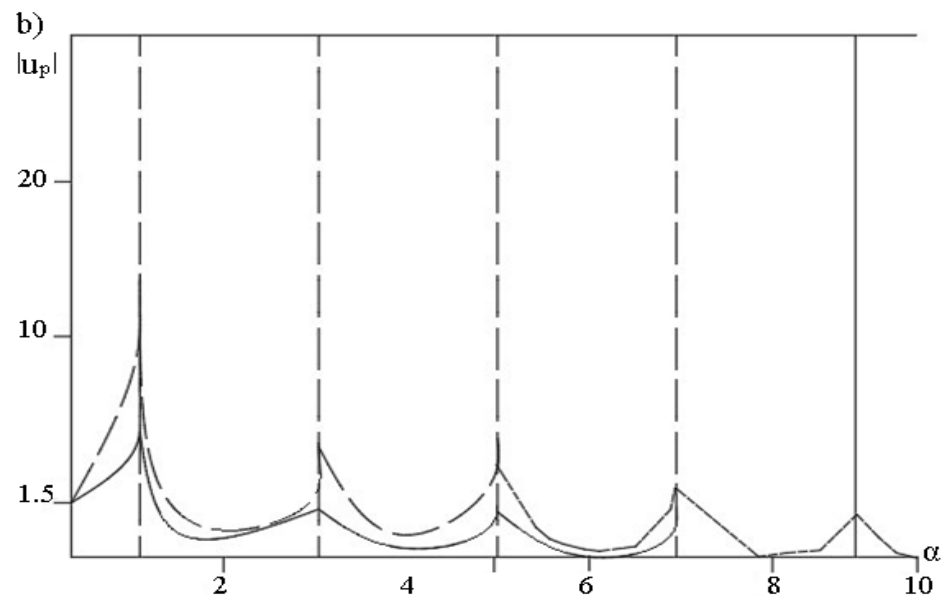

Fig. 4. Amplitude-frequency characteristic of the $\operatorname{rod}$ at $\Omega=5 ; \delta=0.2 ; \mathrm{a}-\eta=0.05 ; \mathrm{b}-\eta=0.10$; $R_{v}=3$.

\section{References}

1. Abduvaliev A A, Abdulkhayzoda A A Transverse vibrations of an underground cylindrical structure. IPICSE (2020) (VII International Scientific Conference INTEGRATION, PARTNERSHIP AND INNOVATION IN CONSTRUCTION SCIENCE AND EDUCATION) DOI.org/10.1088/1757-899X/1030/1/012099

2. Abduvaliev A A, Oscillations of an elastic half-space with a cylindrical. (2016) cavity under the influence of Rayleigh waves. Bulletin of Tashkent Railway Engineering Institute, 4, pp. 38-43.

3. Abduvaliev A. A. Oscillations of a hard disk in elastic space. Bulletin. of TARI, 3, p. 3-10. (2019).

4. Abduvaliev A A, Abdulkhayzoda A A (2020), Underground pipeline damping from the action of Rayleigh waves. CONMECHYDRO-(2020). IOP Conf. Series: Materials Science and Engineering 883 (2020) 012203 doi:10.1088/1757-899X/883/1/012203

5. Abduvaliev A A, Abdulkhayzoda A A Damping vibrations of an underground structure using a three-mass damper. ICECAE (2020). 1st International Conference on Energetics, Civil and Agricultural Engineering (2020), paper 88 IOP Conf. Series: Earth and Environmental Science 614 (2020) 012070 IOP Publishing doi:10.1088/1755-1315/614/1/012070

6. Mirsaidov, M.M., Sultanov, T.Z. Assessment of stress-strain state of earth dams with allowance for nonlinear strain of material and large strains. Magazine of Civil Engineering. 49(5), c. 73-82+136-137. DOI: $10.5862 / \mathrm{MCE} .49 .8$, DOI: 10.5862 MCE.49.8 (2014).

7. Mirsaidov, M.M., Sultanov, T.Z., Rumi, D.F. An assessment of dynamic behavior of the system "structure - Foundation" with account of wave removal of energy. Magazine of Civil Engineering. 39(4), pp. 94-105. DOI: 10.5862/MCE.39.10 (2013).

8. Khodzhaev, D.A., Abdikarimov, R.A., Mirsaidov, M.M. Dynamics of a physically nonlinear viscoelastic cylindrical shell with a concentrated mass, Magazine of Civil Engineering. (2019). 91(7), pp. 39-48. DOI: 10.18720/MCE.91.4

9. Mirsaidov, M., Mekhmonov, Ya. Nonaxisymmetric vibrations of axisymmetric structures with associated masses and hollows (protrusions). Strength of Materials. (1987). 19(3), pp. 424-430. DOI: 10.1007/BF01524147 
10. Mirsaidov, M.M., Toshmatov, E.S. Spatial stress state and dynamic characteristics of earth dams. Magazine of Civil Engineering. (2019). 89(5), pp. 3-15. DOI: 10.18720/MCE.89.1

11. Mirsaidov, M. An account of the foundation in assessment of earth structure dynamics. (2019). E3S Web of Conferences. 97,04015. DOI: $10.1051 / \mathrm{e} 3$ sconf $/ 20199704015$

12. Ishmatov, A.N., Mirsaidov, M. Nonlinear vibrations of an axisymmetric body acted upon by pulse loads. Soviet Applied Mechanics. (1991). 27(4), pp. 388-394. DOI: $10.1007 / \mathrm{BF} 00896519$

13. Mirsaidov, M., Troyanovskii, I.E. Forced axisymmetric oscillations of a viscoelastic cylindrical shell. Polymer Mechanics. 1975. 11(6), pp. 953-955. DOI: $10.1007 / \mathrm{BF} 00857626$

14. Tulkin Mavlanov, Sherzod Khudainazarov and Islomjon Khazratkulov. Natural Vibrations Of Structurally Inhomogeneous Multi-Connected Shell Structures With Viscoelastic Elements. Modelling and Methods of Structural Analysis/ IOP Conf. Series: Journal of Physics: Conf. Series 1425 (2020) 012017 IOP Publishing doi:10.1088/1742-6596/1425/1/012017

15. Korenev B G The method of compensating loads applied to the problems of balance, vibrations and stability of plates and membranes. "Applied Mechanics and Mathematics", v.4, issue 5-6, pp. 61-72. (1940).

16. Pisarenko G.S. Oscillations of mechanical systems with imperfect elasticity of the material. Kiev,-p.379. (1970).

17. Filippov I.G. The effect of viscosity on the propagation of stress waves in elastic bodies under dynamic loads. Applied Mechanics, 15-12, pp. 3-10. (1979).

18. E.S. Sorokin. Method of inelastic resistance of materials when calculating structures for vibrations. In the collection "Research on the dynamics of structures", pp. 5-90. (1951).

19. E.S. Sorokin. Internal friction during vibrations of elastic systems. Gosstroyizdat, p.131. (1980).

20. R. Parner, P. Weidlinger. Dynamic Interaction of a Embedded Cylindrical Rod under Axial Harmonic Forces. International J. Solids Structures, vol. 17, pp. 903-913. (1981). 